\title{
Effects of bone marrow mesenchymal stem cells transplantation on the recovery of neurological functions and the expression of Nogo-A, NgR, Rhoa, and ROCK in rats with experimentally- induced convalescent cerebral ischemia
}

\author{
Jianbo Zhang ${ }^{1}$, Zhenjun $\mathrm{Li}^{1}$, Wenchao Liu ${ }^{1}$, Wenxian Zeng ${ }^{1}$, Chuanzhi Duan ${ }^{1}$, Xuying He ${ }^{1,2}$ \\ ${ }^{1}$ Department of Neurosurgery, The National Key Clinic Specialty, The Neurosurgery Institute of Guangdong Province, Guangdong Province Key \\ Laboratory on Brain Function Repair and Regeneration, Zhujiang Hospital, Southern Medical University, Guangzhou 510282, China; ${ }^{2}$ Department \\ of Neurosurgery, Southern Medical University, Zhujiang Hospital, Guangzhou 510282, China \\ Contributions: (I) Conception and design: X He; (II) Administrative support: X He; (III) Provision of study materials or patients: X He; (IV) Collection \\ and assembly of data: J Zhang, Z Li; (V) Data analysis and interpretation: J Zhang, Z Li; (VI) Manuscript writing: All authors; (VII) Final approval of \\ manuscript: All authors. \\ Correspondence to: Xuying He. Department of Neurosurgery, The National Key Clinic Specialty, The Neurosurgery Institute of Guangdong Province, \\ Guangdong Province Key Laboratory on Brain Function Repair and Regeneration, Zhujiang Hospital, Southern Medical University, Guangzhou \\ 510282, China. Email: 2517079319@qq.com.
}

Background: To investigate the effects of intravenous transplantation of bone marrow mesenchymal stem cells (BMSCs) on neurological function in rats with experimentally-induced convalescent cerebral ischemia and the expression of Nogo-A, NgR, Rhoa, and ROCK expression.

Methods: BMSCs were isolated and cultured in vitro using the whole bone marrow adherent method. Eighty-one adult male Sprague-Dawley rats were divided at random into three groups: the sham-operated group, the cerebral ischemia group, and the BMSC treatment group ( $\mathrm{n}=27$ rats per group). In the latter two groups, the middle cerebral artery occlusion (MCAO) model was performed by the modified Zea Longa method. After MCAO, rats in the sham-operated and cerebral ischemic groups were injected with $1 \mathrm{~mL}$ of phosphate buffered saline (PBS) via the tail vein. In the BMSC-treatment group, $1 \mathrm{~mL}$ of the BMSC suspension (containing $3 \times 10^{6} \mathrm{BMSCs}$ ) was injected through the rats' femoral vein. At 12, 24, and $72 \mathrm{~h}$ after BMSC transplantation, modified neurological deficit scores (mNSS) were used to assess neurological function. TTC (2,3,5-triphenyl tetrazolium chloride) staining was used to measure the ischemic lesion volume, and the distribution of Nogo-A protein was observed by immunohistochemistry. The expressions of Nogo-A, NgR, Rhoa, and ROCK were detected by Western blot.

Results: At $72 \mathrm{~h}$ after BMSC transplantation, the mNSS scores were significantly lower in the BMSC treatment group than those in the cerebral ischemia group $(7.50 \pm 0.55$ vs. $8.67 \pm 0.52, \mathrm{P}<0.01)$, and the ischemic lesions volume was significantly reduced. The expressions of Nogo-A, NgR, RhoA, and ROCK were significantly decreased compared with the controls $(\mathrm{P}<0.05)$.

Conclusions: The transplantation of BMSCs can improve neurological function in rats after convalescent cerebral ischemia, and their therapeutic effect may be related to the downregulation of Nogo-A, NgR, RhoA, and ROCK expression.

Keywords: Bone marrow mesenchymal stem cells (BMSCs); convalescent cerebral ischemia; Nogo-A

Submitted Feb 02, 2020. Accepted for publication Feb 28, 2020.

doi: $10.21037 /$ atm.2020.03.144

View this article at: http://dx.doi.org/10.21037/atm.2020.03.144 


\section{Introduction}

Cerebral ischemia is a common condition with an incidence that is increasing annually and high secondary morbidity and mortality $(1,2)$. Recovery from neurological damage caused by cerebral ischemia is slow. Clinical treatments include antiplatelet aggregation, thrombolysis, and promoting cerebral circulation, although their effects are limited. Therefore, new treatments are urgently needed (3-5).

In recent years, momentum has gathered behind stem cell therapy for the treatment of central nervous system diseases. Bone marrow mesenchymal stem cells (BMSCs) have been reported to play a vital role in regenerative medicine because of their multi-directional differentiation potential and ability to self-replicate. Several experimental animal studies have demonstrated BMSC transplantation to significantly improve neurological function following ischemic injury, promoting nerve repair and improving prognosis (6-9). For the treatment of ischemic injury BMSCs prove effective for several reasons. Firstly, fewer apoptotic cells have been observed to surround lesions after BMSC transplantation into mice. Furthermore, BMSCs have been shown to promote angiogenesis at the ischemic site in the brain and increase blood vessel density $(10,11)$. Secondly, through autocrine or paracrine signaling, BMSCs have been demonstrated to secrete various neuroprotective factors in the brain. These neurotrophic factors induce revascularization in the ischemic region, reduce nerve cell apoptosis, and promote nerve cell survival and regeneration (12-16). These qualities show the value of BMSCs in the treatment of cerebral ischemia.

Central nervous cells, a group of highly differentiated cells, repair and regenerate poorly after cerebral ischemic injury. This is due to their microenvironment after injury as much as it is their low cell regenerative capacity. After cerebral ischemic injury, the microenvironment is rich in various inhibitory factors such as Nogo-A, MAG, and OMgp. The main way Nogo-A hinders axon regeneration is by inhibiting the binding of Nogo receptor and NgR/p75 NTR receptor complex, leading to activation of downstream cascade reaction and the pyramidal degeneration of nerve cell growth, axon atrophy, and axon regeneration failure (17). Among these inhibitory factors, Nogo-A has been reported to play an important role during ischemic brain injury. Nogo-A is in equilibrium with other growth inhibitors and nerve growth promoters. Following cerebral ischemia injury, the internal steady state is destroyed and Nogo-A expression increases so as to inhibit the regeneration and repair of neurons. Nogo-A can activate RhoA and its effect protein kinase (ROCK) by binding to Nogo receptor (NgR), which leads to the collapse of growth cone, thus inhibiting the growth of axon. Some studies have noted that after cerebral ischemia, the expression of Nogo-A was antagonized, and the function of damaged limbs could be recovered obviously, and the plasticity of neurons could be significantly enhanced $(18,19)$. All of this evidence shows that anti-Nogo-A treatment can promote recovery after cerebral ischemic injury $(20,21)$. Therefore, the purpose of this study was to verify the efficacy of BMSCs in treating convalescent cerebral ischemia and to determine whether their effects are mediated through the downregulation of Nogo-A.

\section{Methods}

\section{Animals and groups}

All of the animals in this study were obtained from the Experimental Animal Center of Southern Medical University. Each experiment was conducted in strict accordance with National Institute of Health published guidelines for the welfare and use of experimental animals. Ten one-month-old Sprague Dawley rats weighing 80-100 $\mathrm{g}$ were used for BMSC cultures. Eighty adult male Sprague Dawley rats weighing 280-310 g were used to establish the cerebral ischemia model and to test indicators. During the breeding period, the rats were maintained under conditions of $12 \mathrm{~h}$ of light and $12 \mathrm{~h}$ of dark, at a constant temperature of $25^{\circ} \mathrm{C}$ and a relative humidity of $45-65 \%$. Fresh food and water were provided. The animals were divided into three groups as follows: the sham-operated group (SC, n=27), the cerebral ischemia group (IC, $n=27)$, and the BMSC treatment group (BC, $\mathrm{n}=27)$.

\section{Isolation and cultivation of BMSCs}

Rat bone marrow collection was carried out in strict accordance with the principles of aseptic surgery. Ten rats were sacrificed by cervical dislocation, and then soaked in $75 \%$ ethanol for $10 \mathrm{~min}$. Rat femurs were isolated under aseptic conditions. The muscles, connective tissues, and tendons were removed, and the scaffolds were cut off at both ends. The femoral bone marrow cavity was repeatedly washed with DMEM/F12 (SIGMA, USA). The washed 
cells were centrifuged at $1,000 \mathrm{rpm}$ for $5 \mathrm{~min}$, and the supernatant was discarded. The precipitate was resuspended with DMEM/F12 containing 10\% fetal bovine serum by passing through a pipette tip. Thereafter, the suspension was transferred to a petri dish, marked, and cultured in a humidified $\mathrm{CO}_{2}$ incubator at $37{ }^{\circ} \mathrm{C}$ containing $5 \% \mathrm{CO}_{2}$. The cells were cultured for a period of $8-10$ days and subcultured once they had reached $80-90 \%$ confluence with $2.5 \mathrm{~g} / \mathrm{L}$ trypsin. These steps were repeated until the cells reached the third generation. Thereafter, the morphology of confluent cells was observed under a microscope. BMSCs were collected and suspended in phosphate buffered saline (PBS) to a concentration of $3 \times 10^{6} / \mathrm{mL}$ for further use.

\section{Model preparation}

A rat model of middle cerebral artery occlusion (MCAO) was established using the modified Zea Longa line plug method. A midline carotid incision was made to expose the left common carotid artery (CCA), internal carotid artery (ICA), and external carotid artery (ECA). A traffic branch was created among the electrocoagulation ECA, the ICA ligation, and the free ECA to ensure that the ECA stump length was no less than $0.5 \mathrm{~cm}$. CCA and ICA blood flow was temporarily blocked with a suture. Ophthalmic scissors were used to make a small transverse incision on the ECA stump. The plug from the left ECA stump was inserted into the ICA, and the ECA stump was gently pulled parallel to the ICA. The angle of the line bolt was adjusted so that the horizontal direction of the line bolt was outward, and the horizontal angle was $45^{\circ}$ outward from the midline of the neck. The suture blocking the ICA was removed and gently inserted into the skull. After successful embolization, the ECA stump was ligated, the suture on the CCA was removed, and the incision was closed after no active bleeding was observed. After $2 \mathrm{~h}$ of ischemia, a neck incision was made to pull out the line plug. The shamoperated group underwent the same procedure, except that no wire plug was inserted.

\section{Transplantation procedures}

Three weeks after the establishment of the MCAO model, the BMSC treatment group rats were intravenously injected with $1 \mathrm{~mL}$ of the BMSC cell suspension $\left(3 \times 10^{6}\right.$ cells $/ \mathrm{mL}$ PBS). The cerebral ischemia group rats received an intravenous injection of $1 \mathrm{~mL}$ of $\mathrm{PBS}$, whereas the rats in the surgical group were injected with $1 \mathrm{~mL}$ of PBS postoperatively. All injections were performed immediately after surgery and administered slowly.

\section{Behavioral evaluation}

The neurological function of the rats in each group was evaluated by modified neurological severity scores (mNSS) at 12,24 , and $72 \mathrm{~h}$ after surgery. The mNSS score is a value ranging from 0 to 18 points and takes into account motor, sensory, and reflex indicators. Rats have no associated normal performance and no test reflexes for each score. A higher score indicated a more severe neurological deficit.

\section{Evaluation of infarct volumes}

At 12,24 , and $72 \mathrm{~h}$ after surgery, the brains of the rats in each group were harvested for TTC staining. After anesthesia, the rats were decapitated, and the brain tissues were placed in a refrigerator at $-20{ }^{\circ} \mathrm{C}$ for 20 minutes and then sectioned ( 5 or 6 sections collected every $2 \mathrm{~mm}$ ). The sections were covered with $2 \%$ TTC and placed into an incubator at $37{ }^{\circ} \mathrm{C}$ for $20 \mathrm{~min}$ in the dark. The sections were periodically flipped to ensure even exposure to the staining solution. The sections were then fixed with $0.4 \%$ paraformaldehyde for $24 \mathrm{~h}$. Normal brain tissues stained red, whereas infarcted brain tissues stained white. Images were acquired with a digital camera and analyzed with the Image Pro Plus 6.0 Software.

\section{Western blot analysis}

The brain tissue surrounding the ischemic cortex was removed, homogenized in RIPA buffer, and then centrifuged at $4{ }^{\circ} \mathrm{C}$ for $12,000 \times \mathrm{g}$ for $20 \mathrm{~min}$. The lysed samples were stored at $-80^{\circ} \mathrm{C}$. The BCA assay was applied to quantify the amount of protein. The samples were electrophoresed by $8 \%$ SDS-PAGE for $1.5 \mathrm{~h}$. The proteins were then transferred to PVDF membranes at $300 \mathrm{~mA}$ for $2 \mathrm{~h}$, before blocking with skim milk for $2 \mathrm{~h}$. After this, the blots were incubated with primary antibodies at $4{ }^{\circ} \mathrm{C}$ overnight, followed by the secondary antibody for $2 \mathrm{~h}$. The blots were washed with TBST three times for $30 \mathrm{~min}$ in total after each washing step. The immunoreactive proteins were visualized with ECL reagents using a Bio-Rad 

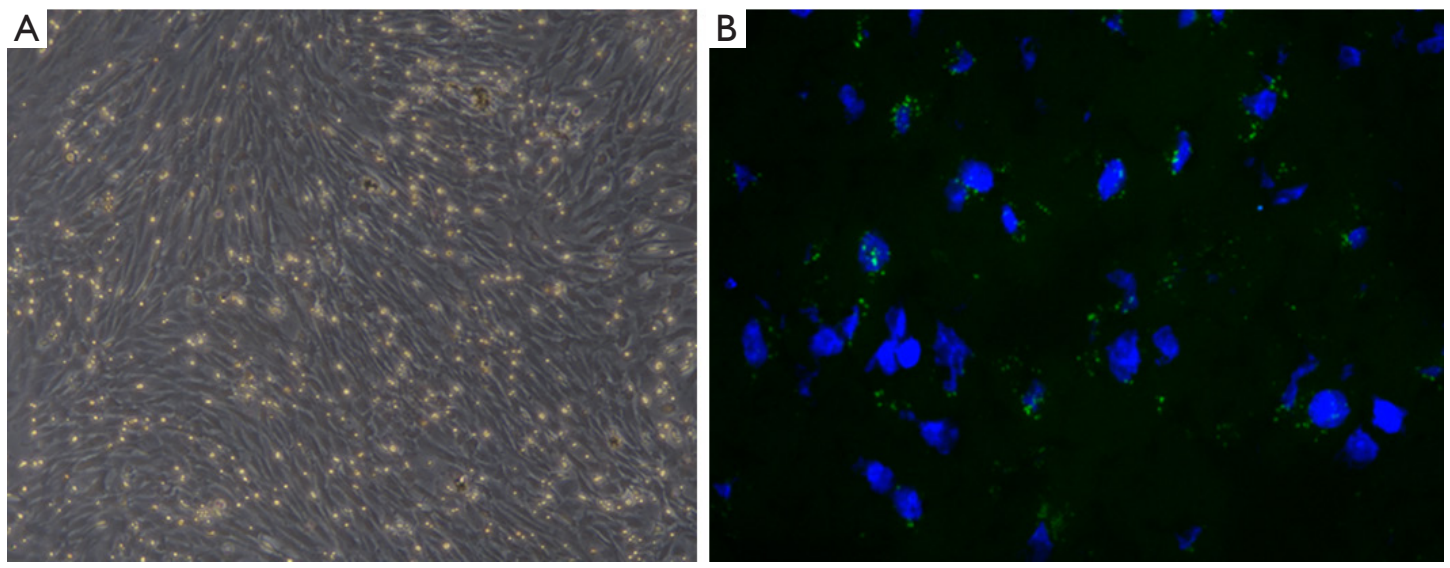

Figure 1 Identification of BMSCs under microscope and representative image of GFP-labeled BMSCs. (A) BMSCs (passage 3) after four days of culture; (B) GFP-labeled BMSCs were observed in the ischemic penumbra at $72 \mathrm{~h}$ after transplantation. BMSC, bone marrow mesenchymal stem cell.

Chemiluminescence System.

\section{Statistical analysis}

SPSS 20.0 software was used for data processing. Measured data with normal distribution were expressed as mean \pm standard deviation $(\mathrm{x} \pm \mathrm{s})$. One-way analysis of variance (ANOVA) was used for comparisons. Pairwise comparison was performed using the LSD-t method. Statistical significance was considered to exist when $\mathrm{P}<0.05$.

\section{Results}

\section{Identification of BMSCs in vitro and the location of BMSCs after transplantation}

The primary cells that had been isolated from the bone marrow were cultured in flasks. Initially, a large number of non-adherent cells were seen under the microscope. After $72 \mathrm{~h}$, the number of adherent cells was significantly increased. The cells were short spindle or polygonal shaped, and those within the colonies were dense. Some apoptotic cells and fragments were visible. On culture days 9 and 10 , the cells were fused in parallel and spread across nearly $90 \%$ of the flask's surface area. The cells displayed whirlpoollike growth and were mostly long spindle shaped. By the third passage, all of the cells were long spindle shaped and displayed vortex-like growth, which is consistent with the morphological characteristics of rat BMSCs (Figure 1A). At $24 \mathrm{~h}$ after transplantation, the infarct lesion in the BMSC treatment group rats was surrounded by GFP-labeled
BMSCs (Figure 1B).

\section{Behavior function after BMSC transplantation}

Modified neurological scores were obtained from rats in the three groups at $12 \mathrm{~h}, 24 \mathrm{~h}$, and $72 \mathrm{~h}$ after surgery (Figure 2). At each time point, the mNSS scores in the cerebral ischemia group were significantly higher than those in the sham-operated group. The scores were lower in the BMSC treatment group than in the cerebral ischemia group, although a statistically significant difference was only noted at $72 \mathrm{~h}(7.50 \pm 0.55$ vs. $8.67 \pm 0.52, \mathrm{P}<0.01)$. There were no significant differences observed in scores between 12 and $24 \mathrm{~h}(12 \mathrm{~h}, 9.50 \pm 0.55$ vs. $9.67 \pm 0.52 ; 24 \mathrm{~h}, 8.50 \pm 0.55$. vs. $9.00 \pm 0.89 ; \mathrm{P}>0.05$ ) (Figure $2 A, B$ ). These findings indicate that MCAO can cause neurological damage in rats, and BMSCs can lessen the functional impairment caused by MCAO.

\section{Infarct volumes after BMSC transplantation}

The cerebral infarct volume was measured by TTC staining (Figure 3A,B). In the sham-operated group, the entire brain stained red, whereas in the cerebral ischemia and BMSC treatment groups, the left brain appeared white. The results showed that the infarct volume was statistically smaller in the brains of the BMSC-treated rats than in the brains of the cerebral ischemic rats. Taken together, these findings indicate that MCAO can cause cerebral infarction, and BMSCs can reduce the extent of cerebral infarction after 


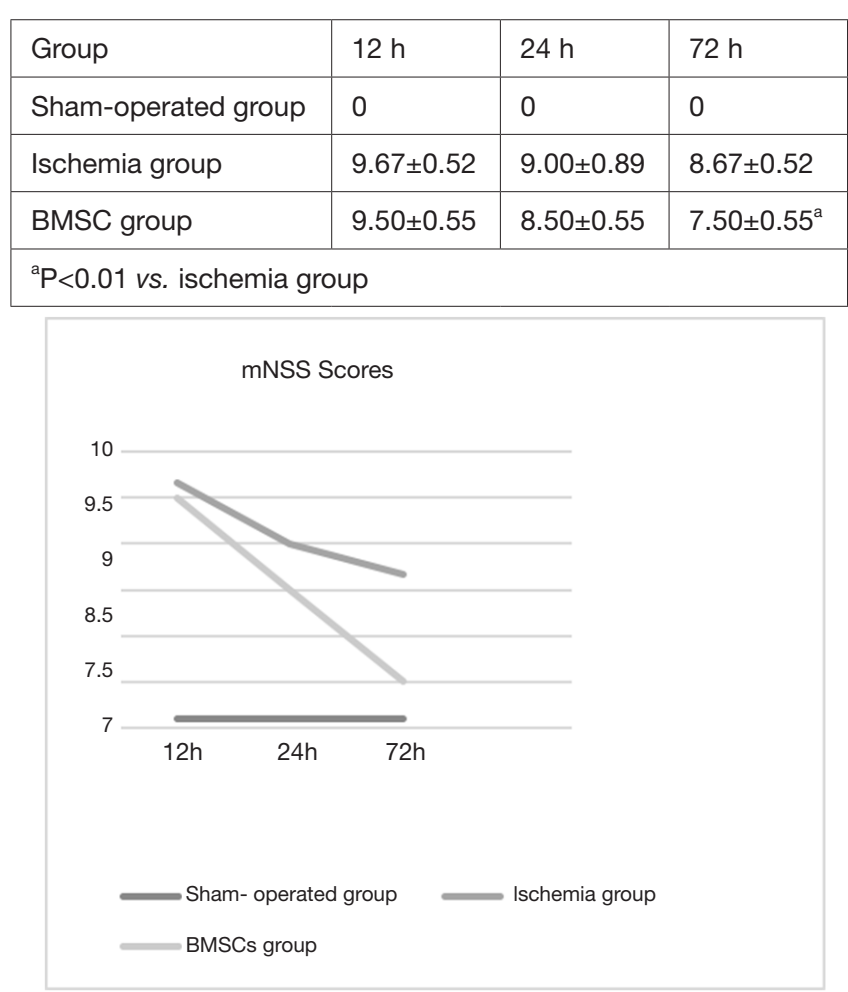

Figure 2 The effect of BMSCs transplantation on neurological function. BMSCs treatment significantly improved neurological function. (A) Comparison of modified neurological severity scale scores after surgery (mean $\pm \mathrm{SD}$ ); (B) comparison of modified neurological severity scale scores in the different groups of rats after operation (mean \pm SD). BMSC, bone marrow mesenchymal stem cell.

treatment.

\section{Expression levels of Nogo-A, NgR, RhoA and ROCK after BMSC transplantation}

At $12 \mathrm{~h}$ after BMSC transplantation, the levels of Nogo-A, $\mathrm{NgR}$, RhoA, and ROCK were significantly higher in the ischemia group than in the sham-operated group. However, the levels of Nogo-A, RhoA, and ROCK were lower in the BMSC treatment group than in the cerebral ischemia group, and $\mathrm{NgR}$ level was not significantly different between the groups. At $24 \mathrm{~h}$, the levels of Nogo-A, NgR, RhoA, and ROCK were lower in the BMSC treatment group than in the cerebral ischemia group. At $72 \mathrm{~h}$, the levels of Nogo-A and RhoA were lower in the cerebral ischemia group than in the BMSC treatment group, and there were no significant differences in the levels of $\mathrm{NgR}$ or ROCK observed between the groups (Figure $4 A, B$ ). These findings indicate that Nogo-A level is significantly elevated in rats after MCAO, and BMSC treatment can downregulate the expression of Nogo-A and its downstream molecules, thereby reducing neurological deficits.

\section{Discussion}

Cerebral ischemia is caused by local blood flow disruption in the brain after cerebral artery occlusion and can results in neurological deficits or death. Neurological deficits after cerebral ischemia include hemiplegia, sensory disturbances, and visual field defects, which indicates the involvement of a complex series of pathophysiological changes. After cerebral ischemia, excitotoxicity, oxidative stress, inflammation, and apoptosis cause severe damage to neurons, glial cells, and vascular endothelial cells, triggering additional events which have further destructive effects through positive feedback mechanisms that eventually lead to neurological deficits and neuronal death.

Synaptic regeneration is a complex process that is limited by the long-term neurological deficits seen following cerebral ischemia. After cerebral ischemic injury, synapse regeneration is affected by a variety of molecular mechanisms, including various neuroprotective or inhibitory factors, of which Nogo-A plays a prominent role. Nogo-A functions in equilibrium with other growthinhibitory and growth-promoting factors. In the presence of an external stimulus, the internal balance is destroyed, and the inhibitory effect of Nogo-A can impede the regeneration of neurons. Previous studies have reported Nogo-A expression to be significantly increased in cerebral ischemic injury, and so the downregulation of Nogo-A expression can markedly promote the regeneration of axons and the recovery of neurological function (21-24). Nogo-A exerts its influence on axon regeneration through binding to multiple receptors such as $\mathrm{NgR}$, PirB, and S1PR2 (25-28). When ischemic cerebrovascular disease occurs, Rho and its downstream effector ROCK are activated in vascular endothelial cells, inflammatory cells and neurons (29). Rock affects the signal transduction system in many ways. It is involved in promoting MLC phosphorylation, increasing the permeability of endothelial cells, and downregulating eNOS expression $(30,31)$. These mechanisms may lead to cerebrovascular blood supply disorder and behavioral dysfunction. Inhibition of the RhoA/ROCK signaling pathway has also been found to promote the induction efficiency of stem cells and to regulate the self-renewal and 


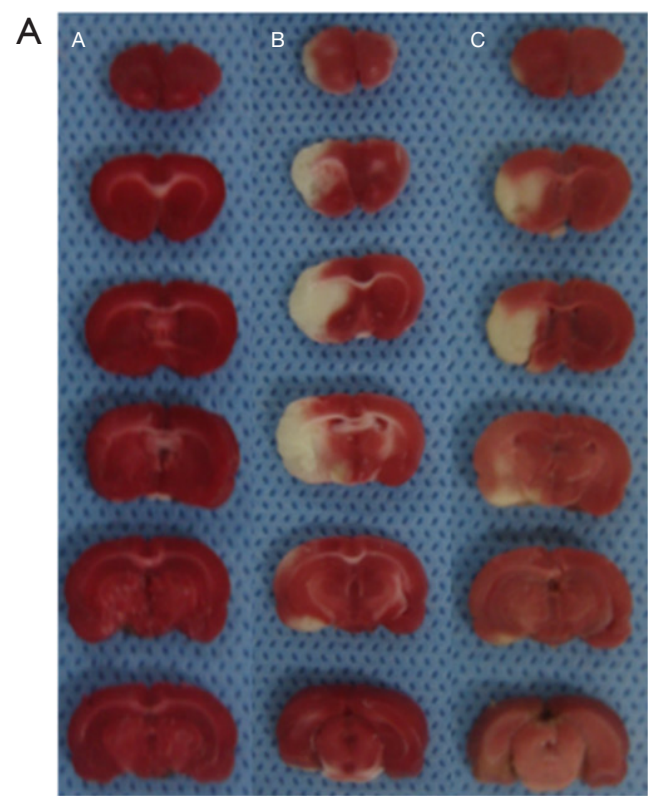

B

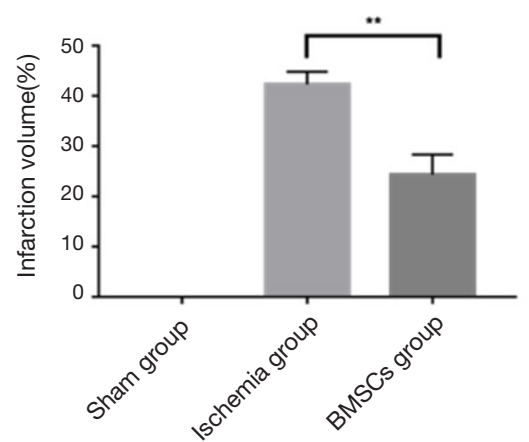

Figure 3 The brain tissue damage of cerebral ischemia rats was weaker in BMSCs group. (A) Cerebral infarction volume as detected by TTC staining; (B) bar graph showing the total brain volume in each group. **, infarction volume. SG, sham-operated group; IG, cerebral ischemia group; BG, BMSC treatment group; BMSC, bone marrow mesenchymal stem cell.

differentiation of stem cells (32). On this basis, the results of our study indicate that BMSC transplantation after cerebral ischemic injury can promote the recovery of neurological function by downregulating the expressions of Nogo-A, NgR, RhoA, and ROCK.

BMSCs are important members of the stem cell family. It originates from the mesoderm in the early stage of development and has the characteristics of multiple differentiation potential and selfreplication. Several experimental animal studies have reported that BMSC transplantation can significantly improve neurological function after ischemic brain injury, promote nerve repair, and improve prognosis (33-37). In this study, 12, 24, and $72 \mathrm{~h}$ after cerebral ischemia were selected as time points for observation, to investigate the neuroprotective mechanism after cerebral ischemia. We found that mNSS scores were lower in the BMSC treatment group than in the cerebral ischemia group, and that the infarct volume was smaller at 12,24 , and $72 \mathrm{~h}$ after BMSC transplantation, indicating that BMSCs can promote nerve repair and improve nerve function in the acute phase. For instance, BMSCs can exert their effects by secreting various neuroprotective factors (e.g., VEGF and bGFG) and growth inhibitory factors (e.g., MAG and OMgp). We also observed that BMSCs can inhibit the expression of Nogo-A. The expression of Nogo-A was significantly increased at $24 \mathrm{~h}$ after cerebral ischemia and slightly decreased at $72 \mathrm{~h}$ after cerebral ischemia. The same observation was made in the shamoperated and BMSC treatment groups. At each of the observation time points after BMSC transplantation, Nogo-A expression was lower in the BMSC treatment group than in the cerebral ischemia group, and its downstream molecules had the same performance, showing that BMSCs exert their therapeutic effects through the inhibition of Nogo-A expression. All of these observations indicate that the body spontaneously recovers after cerebral ischemia, and BMSC can significantly weaken the inhibition of axon growth after cerebral ischemia by downregulating the Nogo-A/NgR/RhoA/ROCK signal pathway. Considering BMSCs can migrate to lesions through transplantation, different rat cells can secrete these factors through various pathways in the brain.

\section{Conclusions}

In summary, BMSC transplantation can effectively promote the recovery of nerve function during the convalescent stage of cerebral ischemia, as well as lower mNSS scores, by downregulating the expressions of Nogo-A, NgR, RhoA, 


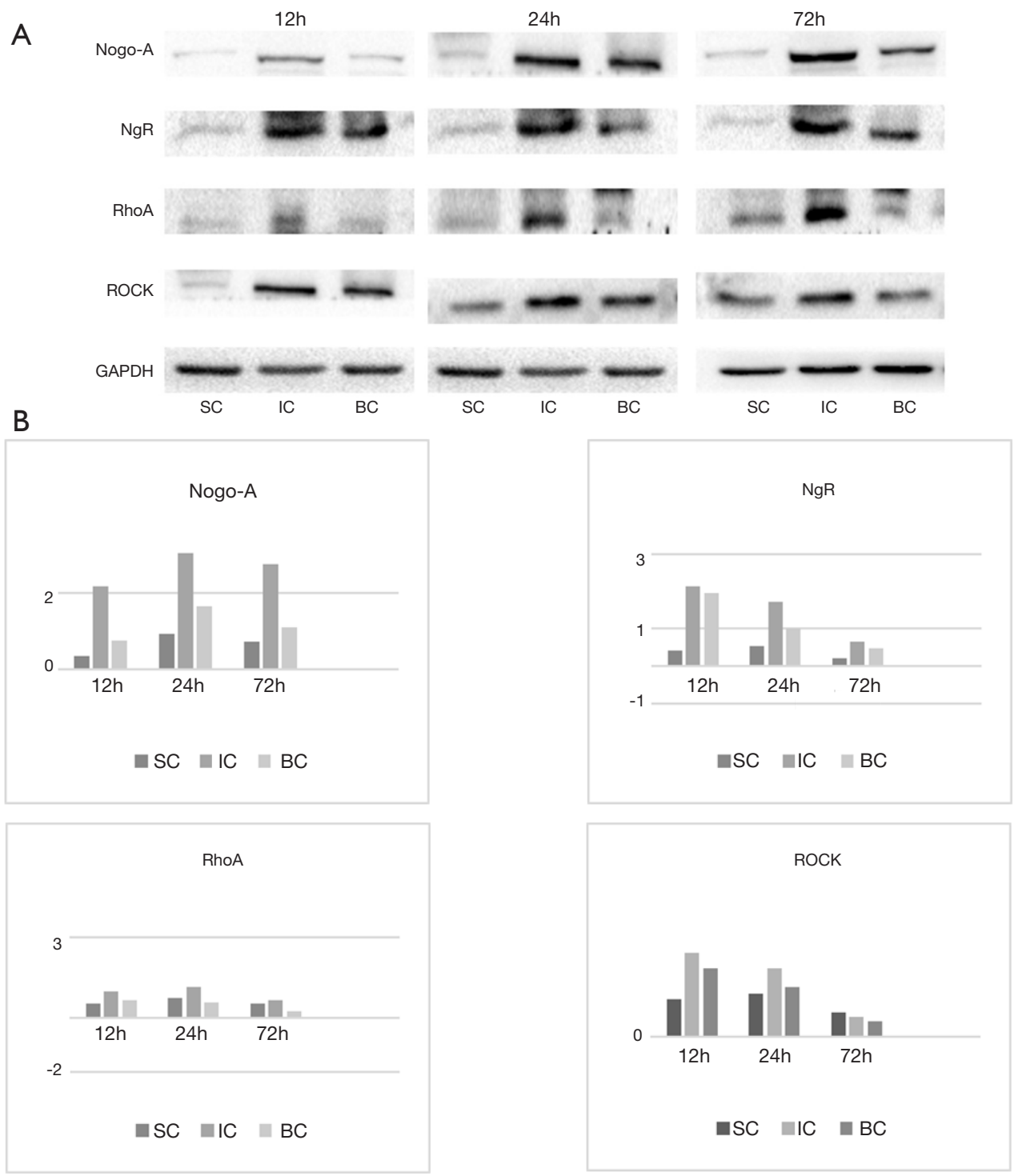

Figure 4 BMSC treatment downregulating the expressions of Nogo-A, NgR, RhoA, and ROCK. (A) The effect of BMSC treatment on the Nogo-A, NgR, RhoA, and ROCK signaling pathway in rats with cerebral ischemic injury. The expressions of Nogo-A, NgR, RhoA, and ROCK in ischemic cerebral tissues at 12, 24, and $72 \mathrm{~h}$ were determined by Western blotting. (B) Relative expression of Nogo-A, NgR, RhoA, and ROCK by western blotting. BMSC, bone marrow mesenchymal stem cell.

and ROCK, and it can also weaken the inhibition of axon regeneration. BMSCs could play an important role in protecting the brain after ischemic stroke. Furthermore, we also determined Nogo-A to be a key factor in the repair of neurological function after cerebral ischemia, which could provide a new possibility for the treatment of cerebral ischemia.

\section{Acknowledgments}

Funding: This study was supported by grants from the Natural Science Funds of China (No. 81400943), 
the National Key Clinical Specialty; the Engineering Technology Research Center of Education Ministry of China, Guangdong Provincial Key Laboratory on Brain Function Repair and Regeneration, and the Department of Neurosurgery, Zhujiang Hospital, Southern Medical University in China.

\section{Footnote}

Conflicts of Interest: The authors have no conflicts of interest to declare.

Ethical Statement: The authors are accountable for all aspects of the work in ensuring that questions related to the accuracy or integrity of any part of the work are appropriately investigated and resolved. All experimental procedures were approved by the Southern Medical University Ethics Committee and were performed in accordance with the guidelines of the National Institutes of Health on the care and use of animals.

Open Access Statement: This is an Open Access article distributed in accordance with the Creative Commons Attribution-NonCommercial-NoDerivs 4.0 International License (CC BY-NC-ND 4.0), which permits the noncommercial replication and distribution of the article with the strict proviso that no changes or edits are made and the original work is properly cited (including links to both the formal publication through the relevant DOI and the license). See: https://creativecommons.org/licenses/by-nc-nd/4.0/.

\section{References}

1. Feigin VL, Norrving B, Mensah GA. Global Burden of Stroke. Circ Res 2017;120:439-48.

2. Feigin VL, Krishnamurthi RV, Parmar P, et al. Update on the Global Burden of Ischemic and Hemorrhagic Stroke in 1990-2013: The GBD 2013 Study. Neuroepidemiology 2015;45:161-76.

3. Catanese L, Tarsia J, Fisher M. Acute Ischemic Stroke Therapy Overview. Circ Res 2017;120:541-58.

4. López-López JA, Sterne J, Thom H, et al. Oral anticoagulants for prevention of stroke in atrial fibrillation: systematic review, network meta-analysis, and cost effectiveness analysis. BMJ 2017;359:j5058.

5. Hankey GJ. Stroke. Lancet 2017;389:641-54.

6. Venkat P, Shen Y, Chopp M, et al. Cell-based and pharmacological neurorestorative therapies for ischemic stroke. Neuropharmacology 2018;134:310-22.

7. Zhang Y, Qiu B, Wang J, et al. Effects of BDNFTransfected BMSCs on Neural Functional Recovery and Synaptophysin Expression in Rats with Cerebral Infarction. Mol Neurobiol 2017;54:3813-24.

8. Li L, Chu L, Fang Y, et al. Preconditioning of bone marrow-derived mesenchymal stromal cells by tetramethylpyrazine enhances cell migration and improves functional recovery after focal cerebral ischemia in rats. Stem Cell Res Ther 2017;8:112.

9. Ye X, Hu J, Cui G. Therapy Effects of Bone Marrow Stromal Cells on Ischemic Stroke. Oxid Med Cell Longev 2016;2016:7682960.

10. Yoo J, Seo JJ, Eom JH, et al. Enhanced recovery from chronic ischemic injury by bone marrow cells in a rat model of ischemic stroke. Cell Transplant 2015;24:167-82.

11. Wu J, Sun Z, Sun HS, et al. Intravenously administered bone marrow cells migrate to damaged brain tissue and improve neural function in ischemic rats. Cell Transplant 2008;16:993-1005.

12. Akhoundzadeh K, Vakili A, Sameni HR, et al. Effects of the combined treatment of bone marrow stromal cells with mild exercise and thyroid hormone on brain damage and apoptosis in a mouse focal cerebral ischemia model. Metab Brain Dis 2017;32:1267-77.

13. He X, Jiang L, Dan QQ, et al. Bone marrow stromal cells promote neuroplasticity of cerebral ischemic rats via a phosphorylated CRMP2-mediated mechanism. Behav Brain Res 2017;320:494-503.

14. Pirzad Jahromi G, Shabanzadeh PA, Sadr SS, et al. Multipotent bone marrow stromal cell therapy promotes endogenous cell proliferation following ischemic stroke. Clin Exp Pharmacol Physiol 2015;42:1158-67.

15. Yang Z, Cai X, Xu A, et al. Bone marrow stromal cell transplantation through tail vein injection promotes angiogenesis and vascular endothelial growth factor expression in cerebral infarct area in rats. Cytotherapy 2015;17:1200-12.

16. Qu R, Li Y, Gao Q, et al. Neurotrophic and growth factor gene expression profiling of mouse bone marrow stromal cells induced by ischemic brain extracts. Neuropathology 2007;27:355-63.

17. Schwab ME. Repairing the injured spinal cord. Science 2002;295:1029-31.

18. Zagrebelsky M, Korte M. Maintaining stable memory engrams: new roles for Nogo-A in the CNS. Neuroscience 2014;283:17-25.

19. Xiao WP, Ding LL, Min YJ, et al. Electroacpuncture 
Promoting Axonal Regeneration in Spinal Cord Injury Rats via Suppression of Nogo/NgR and Rho/ ROCK Signaling Pathway. Neuropsychiatr Dis Treat 2019;15:3429-42.

20. Kucher K, Johns D, Maier D, et al. First-in-Man Intrathecal Application of Neurite Growth-Promoting Anti-Nogo-A Antibodies in Acute Spinal Cord Injury. Neurorehabil Neural Repair 2018;32:578-89.

21. Zemmar A, Weinmann O, Kellner Y, et al. Neutralization of Nogo-A enhances synaptic plasticity in the rodent motor cortex and improves motor learning in vivo. $\mathrm{J}$ Neurosci 2014;34:8685-98.

22. Lindau NT, Banninger BJ, Gullo M, et al. Rewiring of the corticospinal tract in the adult rat after unilateral stroke and anti-Nogo-A therapy. Brain 2014;137:739-56.

23. Jiang W, Xia F, Han J, et al. Patterns of Nogo-A, NgR, and RhoA expression in the brain tissues of rats with focal cerebral infarction. Transl Res 2009;154:40-8.

24. Cheatwood JL, Emerick AJ, Schwab ME, et al. Nogo-A expression after focal ischemic stroke in the adult rat. Stroke 2008;39:2091-8.

25. An H, Brettle M, Lee T, et al. Soluble LILRA3 promotes neurite outgrowth and synapses formation through a high-affinity interaction with Nogo 66. J Cell Sci 2016;129:1198-209.

26. Kempf A, Tews B, Arzt ME, et al. The sphingolipid receptor S1PR2 is a receptor for Nogo-a repressing synaptic plasticity. PLoS Biol 2014;12:e1001763.

27. Gou Z, Mi Y, Jiang F, et al. PirB is a novel potential therapeutic target for enhancing axonal regeneration and synaptic plasticity following CNS injury in mammals. J Drug Target 2014;22:365-71.

28. Huebner EA, Kim BG, Duffy PJ, et al. A multi-domain fragment of Nogo-A protein is a potent inhibitor of cortical axon regeneration via Nogo receptor 1. J Biol Chem 2011;286:18026-36.

Cite this article as: Zhang J, Li Z, Liu W, Zeng W, Duan C, He X. Effects of bone marrow mesenchymal stem cells transplantation on the recovery of neurological functions and the expression of Nogo-A, NgR, Rhoa, and ROCK in rats with experimentallyinduced convalescent cerebral ischemia. Ann Transl Med 2020;8(6):390. doi: 10.21037/atm.2020.03.144
29. Yan YY, Wang XM, Jiang Y, et al. The role of Rho/ Rho-kinase pathway and the neuroprotective effects of fasudil in chronic cerebral ischemia. Neural Regen Res 2015;10:1441-9.

30. Huang L, Li Q, Li H, et al. Inhibition of intracellular $\mathrm{Ca} 2+$ release by a Rho-kinase inhibitor for the treatment of ischemic damage in primary cultured rat hippocampal neurons. Eur J Pharmacol 2009;602:238-44.

31. Popoff MR, Geny B. Multifaceted Role of Rho, Cdc42 and Ras in intercellular junctions, lessions from toxins. Biochim Biophys Acta 2009;1788:797-812.

32. Li Z, Han S, Wang X, et al. Rho kinase inhibitor Y-27632 promotes the differentiation of human bone marrow mesenchymal stem cells into keratinocyte-like cells in xeno-free conditioned medium. Stem Cell Res Ther 2015;6:17.

33. Neal EG, Acosta SA, Kaneko Y, et al. Regulatory T-cells within bone marrow-derived stem cells actively confer immunomodulatory and neuroprotective effects against stroke. J Cereb Blood Flow Metab 2019;39:1750-8.

34. Chau MJ, Deveau TC, Gu X, et al. Delayed and repeated intranasal delivery of bone marrow stromal cells increases regeneration and functional recovery after ischemic stroke in mice. BMC Neurosci 2018;19:20.

35. Morimoto J, Yasuhara T, Kameda M, et al. Electrical Stimulation Enhances Migratory Ability of Transplanted Bone Marrow Stromal Cells in a Rodent Ischemic Stroke Model. Cell Physiol Biochem 2018;46:57-68.

36. He X, Jiang L, Dan QQ, et al. Bone marrow stromal cells promote neuroplasticity of cerebral ischemic rats via a phosphorylated CRMP2-mediated mechanism. Behav Brain Res 2017;320:494-503.

37. Lv B, Li F, Han J, et al. Hif-1alpha Overexpression Improves Transplanted Bone Mesenchymal Stem Cells Survival in Rat MCAO Stroke Model. Front Mol Neurosci 2017;10:80. 